\section{Acquired Extroversion Associated With Bitemporal Variant of Frontotemporal Dementia}

\author{
Mario F. Mendez, M.D., Ph.D. \\ Andrew K. Chen, B.A. \\ Jill S. Shapira, R.N., Ph.D. \\ Po-Huang Lu, Psych.D. \\ Bruce L. Miller, M.D.
}

Frontotemporal dementia (FTD) is characterized by alterations in personality. The nature of the personality changes depend on the localization of the pathology. The authors present three patients with acquired extroversion who met criteria for FTD. All three patients exhibited predominant bilateral temporal involvement as determined with functional neuroimaging. Possible mechanisms for acquired extroversion in bitemporal FTD include bilateral amygdalar damage with decreased interpersonal fear or sense of threat, orbitofrontal-ventromedial damage with social disinhibition, and relative sparing of dorsolateral frontal and anterior cingulate regions with preserved interpersonal agency. FTD can be a vehicle for unraveling the basis of personality characteristics such as the introversion/extroversion dimension.

(The Journal of Neuropsychiatry and Clinical Neurosciences 2006; 18:100-107)

$\mathrm{F}$ rontotemporal dementia (FTD) is the third most common neurodegenerative dementia syndrome after Alzheimer's disease and dementia with Lewy bodies. ${ }^{1,2}$ FTD occurs in about $5 \%$ of memory disorder clinic patients and in a large percentage of dementing diseases that present in the presenium. ${ }^{3-5}$ In the $45-$ to 64 yearold age group, there may be about one patient with FTD for every one patient with early onset Alzheimer's disease. $^{6}$

FTD patients most commonly present with personality changes, and the most common personality changes are apathy, disengagement, and submissiveness. ${ }^{7,8}$ In contrast, we report three patients who presented with acquired extroversion. Most major theories of personality include the dimension of introversion/extroversion, ${ }^{9}$ and these FTD patients offer an opportunity to explore the neurobiological basis of this aspect of personality.

\section{CASE REPORTS}

Case 1

A 66-year-old right-handed, female elementary school teacher, developed a 2-year history of personality change characterized by increasing extroversion and gregariousness. Described as premorbidly shy and retiring, her friends reported that she became talkative with a need to be with and approach other people. She would seek out strangers and initiate conversations, often inappropriately. She would talk to waiters and workmen, such as her gardener, in a flirtatious manner and would stand closer than customary during a conversation, often touching others, and, at one point, forcing candies on them. The patient had also become lighthearted and jocular with frequent punning, joking, smiling, and laughing. When asked about these behaviors, she did not endorse clear changes in her personality. She described herself as "just getting to relax and enjoy her later years." Although the patient appeared overtly aware of others' feelings, she did not express concern when describing her family members with dementia. Finally, her friends described her as newly prone to angry or emotional outbursts and compulsive about collecting cartoons and aphorisms, but they did not observe increased energy or changes in eating and sleeping behavior.

Her medical and psychiatric history was unremarkable except for hypertension, but her family history was strongly positive for early onset dementia. In his late

Received June 14, 2004; revised August 9, 2004; accepted August 16, 2004. From the Departments of Neurology and Psychiatry and Biobehavioral Sciences, University of California Los Angeles, Los Angeles California. Address correspondence to Dr. Mendez, Neurobehavior Unit (116AF), VA Greater Los Angeles Healthcare System, 11301 Wilshire Blvd., Los Angeles, CA 90073; mmendez@ucla.edu (E-mail). Copyright (c) 2006 American Psychiatric Publishing, Inc. 
60s, her father developed a dementing illness with personality changes, and, at about age 60 , both her younger brother and her sister had the onset of a similar decompensation with personality changes. The brother became increasingly jovial with frequent and inappropriate laughter, and her younger sister developed memory and other cognitive deficits and mood lability with pronounced depressive episodes. Both brother and sister developed new-onset alcohol abuse in later life.

On examination, the patient was alert, smiling, and gregarious. Her general behavior was characterized by friendliness, talkativeness, and spontaneous laughter. She ignored personal boundaries, stood too close to her listeners, and often touched them. She had a great many puns and jokes and would burst into laughter over her own humor. Other behavioral observations included a tendency to repeat stereotypical phrases (e.g., "one day at a time" "you are cute"). There was no press of speech, however, and her discourse was easily interruptible. Her thought content was linear without psychotic or obsessional material. The cognitive examination included a Mini-Mental State Examination (MMSE) ${ }^{10}$ score of 24/ 30. She missed three orientation items and three recall items. Language was fluent with good auditory comprehension, repetition, and confrontational naming, and Praxis testing was normal. On a 10-item verbal learning task, she recalled 1 at 15 minutes, and she recalled 9 on the recognition task. Visuospatial constructions were entirely normal, including a clock. She was able to adequately abstract if it was a familiar proverb, but had concrete responses to unfamiliar ones. Other testing showed intact Luria hand sequences. The rest of her neurological examination was normal, including cranial nerves, coordination and motor testing, reflexes, and the sensory examination.

A diagnostic work-up was completed. Her laboratory tests were normal. Neuropsychological testing showed significant impairment in verbal and nonverbal memory with deficits in recognition, minor problems in confrontation naming, impairment in facial recognition, and difficulty with set-shifting and maintenance on a card-sorting task (see Table 1). She could not recognize pictures of famous faces on a 24-item test of well-known American political and entertainment figures. ${ }^{11}$ A close friend completed the Neuropsychiatric Inventory (NPI), ${ }^{12}$ a measure of psychopathology in dementia. On the NPI, the patient was rated as having anxiety, elation, disinhibition, and irritability, all of mild severity except for moderate anxiety. Her MRI was unremarkable, but positron emission tomography (PET) was read as showing bitemporal hypometabolism, greater on the left (see Figure 1).

The patient was diagnosed with FTD, temporal variant. She met established criteria for FTD, ${ }^{13}$ with the exception of more pronounced memory impairment than usual. Her illness was felt to be on a familial, autosomal dominant basis. She was treated with donepezil without definitive response and with possible worsening of her angry, irritable episodes. She was treated with sertraline for her compulsive-like behaviors with a modest response. The patient was followed for 2 years and progressed to naming difficulty of a possible semantic origin and worsening facial recognition. She continued exhibiting extroversion with preservation of visuospatial constructions and other perceptual abilities.

\section{Case 2}

A 57-year-old right-handed woman presented with an 18-month progressive personality change accompanied by difficulty finding words. Her husband complained that she had become uncharacteristically outgoing and loquacious with a tendency to talk to strangers. In his words, his wife had become "the life of the party." She had become childish, with frequent puns, jokes, and bursts of laugher, and disinhibited, with excessively personal or familiar comments about others. She also expressed increased concern over the feelings of others and over their happiness. When asked about the recent death of a favorite uncle, however, the patient expressed verbal sadness but quickly lapsed into laughter, giggling, and a lighthearted responsiveness.

There were other behavioral changes. She had an increasingly empty verbal output with frequent stereotypical phrases. She had decreased self-care with increased sloppiness in dress, often wearing the same clothes over and over again. There was a compulsive tendency, particularly with regard to money, and she would hoard and hide it in different places. She developed an addiction to ice cream and gained a considerable amount of weight. The patient had become anxious and impatient with angry outbursts worsened by donepezil therapy. The patient herself insisted that there was nothing wrong with her and that her "pixy," or Pick's disease, did not affect her in any way. When her specific behaviors were pointed out to her, however, she would say "that is me" and leave it at that.

Her past medical and psychiatric history was unremarkable, but her family history was positive for dementia and amyotrophic lateral sclerosis (ALS). Her pa- 
ternal grandmother developed dementia at age 65 and her paternal uncle had ALS and died at age 58. Her father, however, died at 59 from a stroke.

On examination, the patient was a gregarious woman who continually smiled at the examiner and quickly responded and engaged in conversation. Her MMSE score was 23/30; she missed orientation, attention, and memory items; however, language deficits clearly interfered with her examination. Her spontaneous verbal output had drop out of content words, literal and semantic paraphasias, and stereotypical phrases. She also had a great deal of intrusion from laughter and giggling. She had confrontational naming difficulty and often failed to comprehend the word. The patient comprehended single step and two step commands. Praxis testing was normal. On an auditory verbal learning task, she missed all 10 at 15 minutes, but got 8 on recognition. Her elementary constructions were normal except for the clock drawing, where she put both hands on the one for 10 after 11. Her interpretation of proverbs was concrete, and her other testing showed intact Luria hand sequences. The elementary neurological examination was normal, including cranial nerves, coordination, motor testing, reflexes, and sensory tests.

A diagnostic work-up was undertaken. Neuropsychological testing showed multiple deficits; however, her

\section{TABLE 1. Neuropsychological Tests}

\begin{tabular}{|c|c|c|c|}
\hline Cognitive Domains Assessed & Patient 1 & Patient 2 & Patient 3 \\
\hline \multicolumn{4}{|l|}{ Global Cognitive Functioning } \\
\hline Mini-Mental State Examination & 25 & 23 & 21 \\
\hline Clinical Dementia Rating Scale & 0.5 & 0.5 & 0.5 \\
\hline \multicolumn{4}{|l|}{ Attention } \\
\hline WAIS-III Digit Span & 12 (75th percentile) & 4 (2nd percentile) & 11 (63rd percentile) \\
\hline WMS-III Spatial Span & $\mathrm{N} / \mathrm{A}$ & 3 (1st percentile) & 12 (75th percentile) \\
\hline \multicolumn{4}{|l|}{ Visuospatial Ability } \\
\hline WAIS-III Block Design & 16 (98th percentile) & 3 (1st percentile) & 8 (25th percentile) \\
\hline Rey-Osterrieth Copy & $31 / 36$ (16th percentile) & $\mathrm{N} / \mathrm{A}$ & $\mathrm{N} / \mathrm{A}$ \\
\hline Beery VMI & $\mathrm{N} / \mathrm{A}$ & $6 / 16$ & $15 / 16$ \\
\hline \multicolumn{4}{|l|}{ Remote Memory } \\
\hline WAIS-III Information & 6 (9th percentile) & 3 (1st percentile) & 4 (2nd percentile) \\
\hline Recognition of Famous Faces & $1 / 24$ & $2 / 24$ & $3 / 24$ \\
\hline \multicolumn{4}{|l|}{ Memory } \\
\hline \multicolumn{4}{|l|}{ Verbal Memory } \\
\hline CVLT Short Delay Free Recall* & $0 / 16(<1$ st percentile $)$ & $2 / 9(<1$ st percentile $)$ & $1 / 9$ (2nd percentile) \\
\hline CVLT Long Delay Free Recall* & $0 / 16(<1$ st percentile $)$ & $0 / 9(<1$ st percentile $)$ & $0 / 9$ (2nd percentile) \\
\hline CVLT Long Delay Cued Recall* & $0 / 16$ (<1st percentile) & $2 / 9(<1$ st percentile $)$ & $0 / 9$ (1st percentile) \\
\hline \multicolumn{4}{|l|}{ Visual Memory } \\
\hline WMS-III Visual Reproduction I & 61 (16th percentile) & 0 (<1st percentile) & 39 (2nd percentile) \\
\hline WMS-III Visual Reproduction II & 0 (2nd percentile) & 0 (<1st percentile) & 0 (2nd percentile) \\
\hline \multicolumn{4}{|l|}{ Language } \\
\hline Boston Naming Test (15-Items) & 13 & 8 & 4 \\
\hline Hodges Naming Test & $\mathrm{N} / \mathrm{A}$ & $38 / 64$ & $21 / 64$ \\
\hline FAS & 52 (80th percentile) & 9 (1st percentile) & 14 (4th percentile) \\
\hline Animals & 17 (39th percentile) & 4 (<1st percentile) & 4 (1st percentile) \\
\hline Pyramid and Palm Trees & $\mathrm{N} / \mathrm{A}$ & 40 & 41 \\
\hline \multicolumn{4}{|l|}{ Executive Functions } \\
\hline \multicolumn{4}{|l|}{ Trailmaking Test* } \\
\hline Number Sequencing & 34 (56th percentile) & 150 (<1st percentile) & 54 (10th percentile) \\
\hline Number-Letter Sequencing & 66 (65th percentile) & Discontinued & 199 (5th percentile) \\
\hline \multicolumn{4}{|l|}{ Stroop* $^{*}$} \\
\hline Color & 55 (82nd percentile) & 90 (<1st percentile) & 41 (16th percentile) \\
\hline Word & 42 (63rd percentile) & 47 (<1st percentile) & 29 (25th percentile) \\
\hline Interference & 142 (58th percentile) & Discontinued & $128(<1$ st percentile $)$ \\
\hline \multicolumn{4}{|l|}{ WCST* } \\
\hline Categories & 2 (>16th percentile) & 1 (6-10th percentile) & 0 (2-5th percentile) \\
\hline Perseverative Errors & 19 (37th percentile) & 31 (1st percentile) & $46(<1$ st percentile) \\
\hline Conceptual Level Responses & 63 (19th percentile) & 48 (45th percentile) & 5 (1st percentile) \\
\hline Fail to Maintain Sets & 4 (2-5th percentile) & 0 (>16th percentile) & $0(>16$ th percentile) \\
\hline \multicolumn{4}{|c|}{ * Different versions between patient 1 and patients 2 and 3} \\
\hline \multicolumn{4}{|c|}{ Levels of performance corresponding to the percentiles: 98-99th percentile = very superior; } \\
\hline \multicolumn{4}{|c|}{$91-97$ th percentile $=$ superior; $75-90$ th percentile $=$ high average; $25-74$ th percentile $=$ average; } \\
\hline
\end{tabular}


significant semantic deficits interfered with the tests and their interpretation. She could not recognize pictures of the President of the United States, his wife, other political figures, and several well-known entertainment personalities. On the NPI, ${ }^{12}$ her husband rated her as having mild or moderate agitation, anxiety, elation, apathy, disinhibition, and eating disorders. Her MRI was unremarkable, but her single photon emission computed tomography (SPECT) scan was read as showed bitemporal hypoperfusion, greater on the right (see Figure).

The patient was diagnosed with FTD, ${ }^{13}$ temporal variant. This patient had clear elements of semantic dementia with both word comprehension difficulty and prosopagnosia. Her illness was felt to be on a familial, autosomal dominant basis. She was treated with done- pezil without definitive response and sertraline for compulsive-like behaviors. The patient was followed for 2 years and progressed to dysexecutive performance but with continued extroversion and preservation of visuospatial constructions and other perceptual abilities.

\section{Case 3}

A 71-year-old right-handed woman presented with a 2-year history of gradual and progressive personality change characterized by increasingly outgoing and disinhibited behavior. Her family complained that she approached strangers and initiated conversations, sometimes kissing them in greeting, and rubbing their arms. At one point, she was found sitting on a stranger's lap. She became flip, lighthearted, and silly with frequent laughter and an increased preference for cartoons. She

FIGURE 1. Functional Brain Imaging Studies on the Three Patients
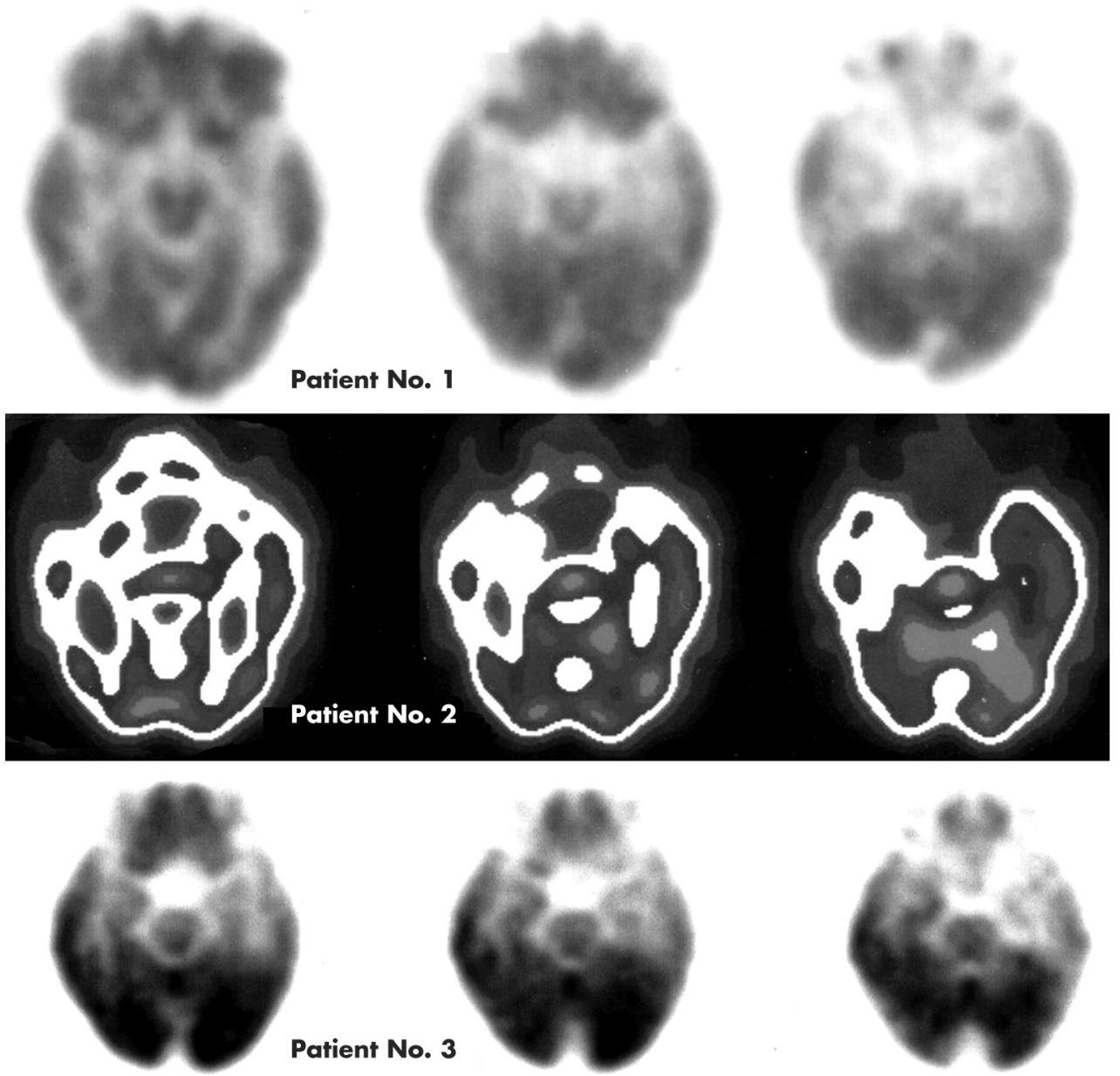

Patients number 1 and 3 underwent positron emission tomography (PET), and patient number 2 underwent single photon emission tomography (SPECT). All three patients were reported to have anterior temporal hypometabolism or hypoperfusion with relative sparing of the frontal lobes. Patients number 1 and 3 had greater involvement on the left than right side, and patient number 2 had greater involvement on the right than the left side. 
verbalized an enhanced expression of love for humanity and become quite generous with her money, giving it away to strangers and others. With time, she became very disinhibited in public, commenting on her sex life and that of her adolescent granddaughter, raising her blouse, touching her granddaughter's breasts, using foul language, and burping or spitting in front of others.

There were other behavioral changes. The patient would repeat the same stereotypical phrases over and over again including "I love you" and "it is a wonder of the world." She had a decline in her eating habits, such as putting her fingers in food at a restaurant or grabbing food with her hands. Her taste in clothes also declined, and she developed compulsive behaviors including the tendency to eat the same foods and hoard money. The patient had become anxious and very impatient, often cutting in front of others in lines, and had occasional angry outbursts made worse by donepezil therapy. Finally, she demonstrated a lack of concern over her husband's severe medical problems.

Her past medical history included a recent face lift, and her family history included a mother and brother with bipolar and another brother with an unknown psychiatric illness. Her family history included several members with new-onset alcoholism in later life.

On examination, the patient was very talkative, but interruptible, with repetitive stereotypical phrases. The patient would run up to the examiners and either hug or kiss them, stand quite close and make endearing or personal comments, or offer them a $\$ 10$ bill. At one point, she became fixated on an examiner's bushy eyebrow and reached up and touched them and, at another point, fondled a medical student's earring. Her MMSE was 27/30; she missed the three memory items only. There was marked anomia on confrontational naming with inability to comprehend the words that she missed. The patient made semantic paraphasic errors. Memory was intact for three of four verbal items at 15 minutes. Praxis was normal. Constructions were normal including the Rey-Osterrieth Complex Figure copy. Abstraction testing tended to the concrete, and she had difficulty with the Luria hand sequences. Her neurological examination was otherwise intact including cranial nerves, gait, station, motor exam, reflexes, and sensation.

A diagnostic work-up was initiated. Routine laboratory work was normal. Her neuropsychological testing showed impairments in language, memory, and executive functions (see Table 1). On the NPI, her husband rated her as having mild or moderate anxiety, disinhibition, irritability, aberrant motor behavior, sleep disor- ders, and eating disorders. Similar to the two prior patients, she could not recognize pictures of famous faces. Her MRI was unremarkable, but a PET showed bitemporal hypoperfusion, worse on the left (see Figure 1).

The patient was diagnosed with $\mathrm{FTD}^{13}{ }^{13}$ temporal variant possibly on a familial basis. Treatment was tried with a number of psychoactive medications, including clomipramine and aripiprazole. Her behavioral disturbances were eventually managed with quetiapine and paroxetine. After 2 years of follow up, the patient continued to lose semantic information and continued to be extroverted and disinhibited.

\section{DISCUSSION}

We report three patients with acquired extroversion who had a bitemporal variant of FTD. They met Consensus Criteria for FTD with an insidious onset and progressive disorder characterized by alterations in social interpersonal behavior, decreased personal regulation, and decreased insight. ${ }^{13}$ Although they did not have emotional blunting in interpersonal interactions, they did not respond with appropriate concern for their family members. All three had disinhibition, puerile behavior with laughter and punning, and angry emotional outbursts made worse by an acetylcholinesterase inhibitor in two. Finally, all three had facial recognition difficulty and two had semantic deficits suggesting semantic dementia.

The central behavioral change in these patients was more consistent with acquired extroversion rather than just social disinhibition. Extroversion refers to a personality dimension where a person's energies are directed toward other people and to the outer world. ${ }^{9}$ These three patients were not just disinhibited on encountering others. They actively sought social engagements as well as new experiences, whereas, prior to developing FTD, they were more solitary and cautious.

In FTD, personality changes are the most prominent symptoms and usually precede or overshadow their cognitive disabilities. ${ }^{4,5,14,15}$ FTD patients often become inactive with decreased behavioral initiation and spontaneity. ${ }^{7,16}$ This reflects increased submissiveness, decreased social dominance, and apathy from pathology in the dorsolateral frontal lobes and anterior cingulate. ${ }^{7,8}$ FTD patients may also have poor social awareness or disinhibition, loss of insight, stereotypical or repetitive behaviors, hyperoral or other eating behavior changes, and self-neglect in the face of relatively preserved memory and visuospatial ability. ${ }^{17-20}$ In addition, they can 
lack empathy and lose the ability to infer other people's mental states, thoughts, and feelings. ${ }^{21-23}$

The onset of FTD is frequently asymmetric and may be predominantly a temporal variant. The usual pathology in the "temporal" variant involves atrophy of the amygdala and the anterior temporal lobes and extends to include the orbitofrontal-ventromedial frontal cortex..$^{24}$ These patients often have semantic anomia and impaired visual recognition, particularly for faces. ${ }^{13}$ The perception of facial or vocal emotions and affect may be deficient, $^{22,25}$ especially anger, sadness, and fear in those with right temporal lobe involvement. ${ }^{22,25-28}$ Right temporal patients are particularly behaviorally disturbed with irritability, disinhibition, and a loss of empathy and interpersonal warmth. ${ }^{8,29-32}$ Although the nomenclature is somewhat controversial, the literature often refers to FTD patients with predominant temporal involvement as having "semantic dementia" when the cognitive aspects predominate and "FTD-temporal variant" when the behavioral aspects predominate. ${ }^{13,29}$

FTD-temporal variant patients may be informative about the neurobiology of extroversion. Similar to these patients, extroverts have lower cerebral blood flow in the temporal lobes compared to introverts on single photon emission computed tomography. ${ }^{33}$ FTD-temporal variant patients also resemble the neurobiologically based hypersociability of William's syndrome, from an interstitial deletion of chromosome 7. ${ }^{34,35}$ Extroversion relies on several underlying neurobiological mechanisms. ${ }^{9}$ One of these is a drive toward interpersonal contact rather than to avoidance or withdrawal from others. Another is increased impulsivity or decreased social constraints. Extroversion also requires interpersonal agency or a motivation to be socially dominant, assertive, and exhibitionistic. $^{9}$

Damage to the amygdala may promote interpersonal contact from decreased social apprehension. The amygdala processes perception of emotional cues and produces emotional responses, especially those that are fear-related or involved in avoidance-withdrawal. Bilateral amygdalar involvement can result in decreased fear, especially from faces, and the tendency to judge others as more trustworthy and approachable. ${ }^{36,37}$ Mature rhesus monkeys with bilateral lesions of the amygdala engage in more affiliative social interactions with decreased inhibitions. ${ }^{38}$ In comparison, patients with William's syndrome have larger amygdala. ${ }^{35}$ suggesting that the amygdalar effects on extroversion vary depending on whether the changes are congenital or involve specific parts of the amygdala.
Compared to introverts, extroverts have less social inhibition and may be expected to have lower activity in behavioral inhibition systems in the orbitofrontal-ventromedial region ${ }^{39,40}$ — areas damaged in FTD-temporal variant. Among normals, introverts have high cortical activity in the frontal lobes from activities mediated, at least in part, by orbitofrontal and ventromedial frontal cortex. ${ }^{40}$ With the amygdala, the orbitofrontal cortex processes the reward or punishment value of emotionally salient stimuli. With the ventromedial frontal cortex, the orbitofrontal cortex processes the emotional and social significance of perceptions and triggers a reenactment of previously encountered emotional experiences. Injury to the orbitofrontal-ventromedial region impairs social judgment and facilitates the social disinhibition present in these FTD-temporal variant patients.

In contrast, these patients have relative sparing of the dorsolateral frontal cortex and the anterior cingulate gyrus with retained interpersonal agency. There is less frontal apathy or passive submissiveness and loss of social dominance in the FTD-temporal variant, as opposed to the FTD-frontal variant. ${ }^{8}$ The dorsolateral frontal cortex represents the goal states toward which patients are directed, and the anterior cingulate represents the attentional effects of emotional arousal, emotional responses, and motor response initiation. ${ }^{41}$ Extroverted people show a higher level of activation of the anterior cingulate cortex compared with introverted people, ${ }^{40}$ and patients with William's syndrome seem to have large anterior cingulate gyri. ${ }^{35}$

There are other potential contributing factors for extroversion in FTD. Extroversion depends on an intact incentive motivation circuit composed of the nucleus accumbens, ventral pallidum, ventral tegmental area, and their dopaminergic projections to the frontal lobes. ${ }^{9}$ Injury to the insula could play a role through decreased visceral representations and internal representations, ${ }^{40}$ For example, blood flow in the anterior insula correlates with introversion, consistent with recalling or imagining events. ${ }^{40}$ Finally, extroversion may depend on the relative bilateral involvement of the right and left hemispheres. In FTD, specific behaviors may arise from balanced mechanisms involving both hemispheres. ${ }^{42}$ Bilateral involvement in FTD may explain the difference between these extroverted patients and those with more unilateral right temporal involvement who lose empathy and emotional warmth. ${ }^{8,32}$ Moreover, other work indicates that manic-type behavior with apparent extroversion can result from bitemporal abnormalities. ${ }^{43}$ 
This preliminary clinical report suggests directions for future research and raises further questions. First, it recommends a prospective study on the nature of extroversion in FTD. Second, it raises questions about the role of anxiety in the patients' frantic need to approach and interact with others. Although family and friends viewed these patients as highly anxious, the patient's did not describe themselves as experiencing anxiety, panic, or nervousness. Finally, an incidental observation not clearly related to extroversion is the presence of memory loss. As FTD progresses, memory is eventually compromised, but there is relative preservation of recognition memory compared to free recall. ${ }^{44,45}$ Occasionally, FTD patients have more severe memory impairment, ${ }^{46}$ as in our patients, and this may indicate greater temporal lobe involvement in FTD.

\section{CONCLUSION}

Bitemporal FTD may result in acquired extroversion. Three proposed factors include 1) affiliation or decreased avoidance-withdrawal from biamygdalar disease, 2) social disinhibition from orbitofrontal-ventromedial involvement, and 3) retained agency or social dominance from relative sparing of dorsolateral frontal cortex and anterior cingulate gyrus. Significant research will be needed in the future to determine the precise mechanisms of introversion/extroversion in brain diseases such as FTD.

Research for this study was supported in part by the $\mathrm{Na}$ tional Institute on Aging (NIA) (grant AG-19724-01), the University of California Los Angeles Alzheimer's Disease Center, and the State of California.

\section{References}

1. Kertesz A, Munoz DG: Frontotemporal dementia. Med Clin North Am 2002; 86:501-518

2. Pasquier F, Petit H: Frontotemporal dementia: its rediscovery. Eur Neurol 1997; 38:1-6

3. Gustafson L, Brun A, Passant U: Frontal lobe degeneration of non-Alzheimer type. Baillieres Clin Neurol 1992; 1:559-582

4. Pasquier F, Delacourte A: Non-Alzheimer degenerative dementias. Curr Opin Neurol 1998; 11:417-427

5. The Lund and Manchester Groups: Clinical and neuropathological criteria for frontotemporal dementia. J Neurol Neurosurg Psychiatry 1994; 57:416-418

6. Ratnavalli E, Brayne C, Dawson K, Hodges JR: The prevalence of frontotemporal dementia. Neurol 2002; 58:1615-1621

7. Liu W, Miller BL, Kramer JH, et al: Behavioral disorders in the frontal and temporal variants of frontotemporal dementia. Neurol 2004; 62:742-748

8. Rankin KP, Kramer JH, Mychack P, et al: Double dissociation of social functioning in frontotemporal dementia. Neurol 2003; 60:266-271

9. Depue RA, Collins PF: Neurobiology of the structure of personality: dopamine, incentive motivation and extraversion. Behav Brain Sci 1999; 22:491-569

10. Folstein MF, Folstein SE, McHugh PR: Mini-mental state: a practical method for grading the cognitive state of patients for the clinician. J Psychiatr Res 1975; 2:189-198

11. Mendez MF, Ghajarani M: Agnosia for familiar faces and odors in a patient with right temporal lobe dysfunction. Neurol 2001; 57:519-521

12. Cummings JL: The Neuropsychiatry Inventory: assessing psychopathology in dementia patients. Neurol 1997; 48(5 Suppl 6):S10-S16

13. Neary D, Snowden JS, Gustafson L, et al: Frontotemporal lobar degeneration: a consensus on clinical diagnostic criteria. Neurol 1998; 51:1546-1554

14. Mendez MF, Selwood A, Mastri AR, et al: Pick's disease versus
Alzheimer's disease: a comparison of clinical characteristics. Neurology 1993; 43:289-292

15. Miller BL, Cummings JL, Villanueva-Meyer J, et al: Frontal lobe degeneration: clinical, neuropsychological, and SPECT characteristics. Neurol 1991; 41:1374-1382

16. Galante E, Muggia S, Spinnler H, et al: Degenerative dementia of the frontal type: clinical evidence from 9 cases. Dement Geriatr Cogn Disord 1999; 10:28-39

17. Bathgate D, Snowden JS, Varma A, et al: Behaviour in frontotemporal dementia, Alzheimer's disease and vascular dementia. Acta Neurol Scand 2001; 103:367-378

18. Bozeat S, Gregory CA, Ralph MA, et al: Which neuropsychiatric and behavioural features distinguish frontal and temporal variants of frontotemporal dementia from Alzheimer's disease? J Neurol Neurosurg Psychiatry 2000; 69:178-186

19. Gustafson L: Clinical picture of frontal lobe degeneration of nonAlzheimer type. Dementia 1993; 4:143-148

20. Mendez MF, Cherrier M, Perryman KM, et al: Frontotemporal dementia versus Alzheimer's disease: differential cognitive features. Neurol 1996; 47:1189-1194

21. Gregory C, Lough S, Stone V, et al: Theory of mind in patients with frontal variant frontotemporal dementia and Alzheimer's disease: theoretical and practical implications. Brain 2002; 125:752-764

22. Lavenu I, Pasquier F, Lebert F, et al: Perception of emotion in frontotemporal dementia and Alzheimer disease. Alzheimer Dis Assoc Disord 1999; 13:96-101

23. Lough S, Gregory C, Hodges JR: Dissociation of social cognition and executive function in frontal variant frontotemporal dementia. Neurocase 2001; 7:123-130

24. Rosen HJ, Gorno-Tempini ML, Goldman WP, et al: Patterns of brain atrophy in frontotemporal dementia and semantic dementia. Neurol 2002a; 58:198-208

25. Keane J, Calder AJ, Hodges JR, et al: Face and emotion process- 
ing in frontal variant frontotemporal dementia. Neuropsychologia 2002; 40:655-665

26. Perry RJ, Miller BL: Behavior and treatment in frontotemporal dementia. Neurol 2001; 56:S46-S51

27. Rosen HJ, Perry RJ, Murphy J, et al: Emotion comprehension in the temporal variant of frontotemporal dementia. Brain 2002b; 125:2286-2295

28. Rosen HJ, Pace-Savitsky K, Perry RJ, et al: Recognition of emotion in the frontal and temporal variants of frontotemporal dementia. Dement Geriatr Cogn Disord 2004; 17:277-281

29. Edwards-Lee T, Miller BL, Benson DF, et al: The temporal variant of frontotemporal dementia. Brain 1997; 120:1027-1040

30. Mendez MF, Chow T, Ringman J, et al: Pedophilia and temporal lobe disturbances. J Neuropsychiatry Clin Neurosci 2000; 12:7176

31. Mychack P, Kramer JH, Boone KB, et al: The influence of right frontotemporal dysfunction on social behavior in frontotemporal dementia. Neurol 2001; 56:S11-S15

32. Perry RJ, Rosen HR, Kramer JH, et al: Hemispheric dominance for emotions, empathy and social behaviour: evidence from right and left handers with frontotemporal dementia. Neurocase 2001; 7:145-160

33. Stenberg G, Risberg J, Warkentin S, Rosen I: Regional patterns of cortical blood flow distinguish extraverts from introverts. Personality Indiv Differ 1990; 11:663-673

34. Doyle TF, Bellugi U, Korenberg JR, et al: "Everybody in the world is my friend" hypersociability in young children with Williams syndrome. Am J Med Genet 2004;124A:263-273

35. Reiss AL, Eckert MA, Rose FE, et al: An experiment in nature: brain anatomy parallels cognition and behavior in Williams syndrome. Neurobiol Dis 2004; 24:5009-5015

36. Adolphs: The neurobiology of social cognition. Cur Opin Neurobiol 2001; 11:231-239

37. Anderson AK, Spencer DD, Fulbright RK, et al: Contribution of the anteromedial temporal lobes to the evaluation of facial emotion. Neuropsychology 2000; 14:526-536

38. Amaral DG: The amygdala, social behavior, and danger detection. Ann NY Acad Sci 2003; 1000:337-347

39. Gray JA: The psychophysiological basis of introversion-extraversion. Behav Res Ther 1970; 8:249-266

40. Johnson DL, Wiebe JS, Gold SM, et al: Cerebral blood flow and personality: A positron emission tomography study. Am J Psychiatry 1999; 156:252-257

41. Davidson RJ, Irwin W: The functional neuroanatomy of emotion and affective style. Trends Cogn Sci 1999; 3:11-21

42. Rankin KP, Rosen HJ, Kramer JH, et al: Right and left medial orbitofrontal volumes show an opposite relationship to agreeableness in FTD. Dement Geriatr Cogn Disord 2004; 17:328-332

43. Carran MA, Kohler CG, O'Connor MJ, et al: Mania following temporal lobectomy. Neurol 2003; 61:770-774

44. Glosser G, Gallo JL, Clark CM, Grossman M: Memory encoding and retrieval in frontotemporal dementia and Alzheimer's disease. Neuropsychology 2002; 16:190-196

45. Pasquier F, Grymonprez L, Lebert F, et al: Memory impairment differs in frontotemporal dementia and Alzheimer's disease. Neurocase 2001; 7:161-171

46. Caine D, Patterson K, Hodges JR, et al: Severe anterograde amnesia with extensive hippocampal degeneration in a case of rapidly progressive frontotemporal dementia. Neurocase 2001; 7:57-64 